\title{
Knockdown of DUXAP10 inhibits proliferation and promotes apoptosis in bladder cancer cells via PI3K/Akt/mTOR signaling pathway
}

\author{
XIU-YI LV ${ }^{1}$, LIANG MA ${ }^{1,2}$, JUN-FENG CHEN ${ }^{1}$, RUI YU ${ }^{3}$,

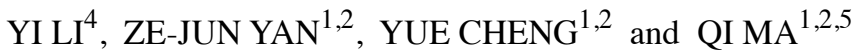

\begin{abstract}
${ }^{1}$ Translational Research Laboratory for Urology, Key Laboratory of Ningbo, ${ }^{2}$ Department of Urology, Ningbo First Hospital, The Affiliated Hospital of Ningbo University, Ningbo, Zhejiang 315010; ${ }^{3}$ Department of Biochemistry and Molecular Biology, Zhejiang Key Laboratory of Pathophysiology, School of Medicine, Ningbo University, Ningbo, Zhejiang 315211; ${ }^{4}$ Department of Urology, The Second Affiliated Hospital of Zhejiang University, School of Medicine, Hangzhou, Zhejiang 310009; ${ }^{5}$ Department of Urology, The Ninth Hospital of Ningbo, Ningbo, Zhejiang 315020, P.R. China
\end{abstract}

Received August 2,2017; Accepted October 24, 2017

DOI: $10.3892 /$ ijo.2017.4195

\begin{abstract}
DUXAP10 is a member of long non-coding RNAs (lncRNAs) and has been reported to be upregulated in bladder cancer (BC) tissues. However, the biological functions of DUXAP10 in BC are largely unknown. The present study detected the expression of DUXAP10 in human normal bladder cell SV-HUC-1 and BC cell lines. Subsequently, cell proliferation, cell cycle, and apoptosis were analyzed by knockdown of the DUXAP10 expression. Results suggested that the expression level of DUXAP10 was significantly enhanced in cancer cells. After knockdown of DUXAP10, cell proliferation was inhibited, cell cycle was arrested at G0/G1 phase, and apoptosis was increased in T24 and 5637 cells. Western blot analysis detected that knockdown of DUXAP10 decreased the expression of Bcl-xL, cyclin D and CDK4. This increased the expression of Bad, cleaved caspase-3, cleaved caspase-9, and p27. Further studies indicated that knockdown of DUXAP10 inhibited PI3K/Akt/mTOR signaling pathway. Combining these results, our study suggests that DUXAP10 plays an important role in BC and DUXAP10 inhibition is a potential therapeutic target for $\mathrm{BC}$.
\end{abstract}

\section{Introduction}

Bladder cancer (BC) is the most common malignancy of the urinary tract and is associated with significantly high morbidity

Correspondence to: Professor Yue Cheng or Professor Qi Ma, Translational Research Laboratory for Urology, Key Laboratory of Ningbo, Ningbo First Hospital, The Affiliated Hospital of Ningbo University, 59 Liuting Street, Ningbo, Zhejiang 315010, P.R. China

E-mail: yue.cheng@yahoo.com

E-mail: qi_ma@yahoo.com

Key words: DUXAP10, bladder cancer, cell proliferation, cycle cycle, apoptosis, PI3K/Akt/mTOR signaling pathway and mortality $(1,2)$. BC can be divided into three groups: nonmuscle invasive $\mathrm{BC}$ (NMIBC), muscle-invasive $\mathrm{BC}(\mathrm{MIBC})$ and metastatic BC. Approximately $20-25 \%$ of patients were found to have MIBC and the remaining $75-80 \%$ of patients had NMIBC at the first diagnosis (3). Transurethral resection of bladder tumor (TRUBT) combined with intravesical chemotherapy is typically the primary method for the treatment of NMIBC (4). Once BC invades muscle, radical cystectomy should be performed to improve the treatment success rate (5). Due to current surgical limitations in radical cystectomy, up to $50 \%$ of MIBC patients will eventually experience recurrence and progress to metastatic disease (3). If metastatic $\mathrm{BC}$ presents, systemic chemotherapy and PD-1/PD-L1 antibody based immunotherapy are the primary choices to prolong the quantity and quality of life, although the majority of patients may eventually die of their disease $(6,7)$. These statistics demonstrates the urgent necessity for the discovery of new biomarkers to detect $\mathrm{BC}$, as well as the need to produce new pharmaceutical solutions for the treatment of BC.

Recent data obtained from the ENCODE consortium indicate that $\sim 70 \%$ of the human genome is transcribed, generating a vast range of non-coding RNAs (8). Long noncoding RNAs (lncRNAs) are a class of RNA molecules defined as transcripts longer than 200 nucleotides, although these molecules lack protein-coding potential. lncRNAs are found in sense or antisense orientation for protein-coding genes, within introns of protein-coding genes or intergenic regions of the genome (9). Though small non-coding RNAs (<200 bp), particularly miRNAs (21-23 nucleotides), are well characterized as post-transcriptional regulators of mRNAs and their roles in cancer are well established $(10,11)$, lncRNAs are still under investigation and more information is needed to understand the role of lncRNAs in cancer occurrence and progression (12).

Several lncRNAs have been identified for their relation to BC. For example, UCA1 was demonstrated to be highly expressed in the $\mathrm{BC}$ tissues, and promote glutamine metabolism by targeting miR-16 in human BC (13). ANRIL was 
reported to be significantly overexpressed in $\mathrm{BC}$ tissues and regulated $\mathrm{BC}$ cell proliferation and apoptosis both in vitro and in vivo (14). GHET1 was demonstrated to be significantly upregulated in $\mathrm{BC}$ tissues and promoted the proliferation and invasion of BC cells (15). These results indicate that lncRNAs can be a possible source of valuable information to better understand the potential mechanisms underlying $\mathrm{BC}$ occurrence and progression.

DUXAP10 is a newly found lncRNA, the expression of which was significantly elevated in BC tissues compared to matched normal bladder tissues ( $>8$-fold changes) by lncRNA microarray (16). However, its biological functions in $\mathrm{BC}$ are largely unknown. To further investigate its role in BC cells, we first detected the expression level of DUXAP10 in human normal bladder epithelial cell line SV-HUC-1 and different BC cell lines. T24 and 5637 were then selected as a potential cell model for further investigation. Specific siRNA oligos targeting DUXAP10 (si-DUXAP10) were transfected to T24 and 5637 cells, and cell proliferation, apoptosis and cell cycle changes were observed.

\section{Materials and methods}

Cell culture. Human BC cell lines (5637, T24, E-j, TCCSUP, UM-UC-3, RT4) and human normal bladder epithelial cell line SV-HUC-1 were obtained from the Institute of Cell Research, Chinese Academy of Sciences, Shanghai, China. The human $\mathrm{BC}$ cell lines and normal bladder epithelial cell line were respectively cultured in RPMI-1640 (5637, T24, E-j), MEM (TCCSUP, UM-UC-3), McCoy's 5A Medium (RT4) (Gibco, Life Technologies, Carlsbad, CA, USA) and F12-k medium (Gibco). All media were supplemented with $10 \%$ fetal bovine serum (FBS; Gibco) and 1\% penicillin-streptomycin (Gibco). All cell lines were cultured in a humidified air atmosphere of $5 \% \mathrm{CO}_{2}$ at $37^{\circ} \mathrm{C}$. Cell transfection was conducted by Lipofectamine 2000 .

Total RNA extraction and reverse transcription. Total RNA from cells was extracted using TRIzol reagent (Invitrogen, Karlsruhe, Germany) following the manufacturer's instructions. Subsequently, total RNA was quantified using a NanoDrop ND-2000 (Thermo Fisher Scientific, Wilmington, DE, USA). cDNA was synthesized by reverse transcription (RT) using random primers and the GoScript RT system (Promega, Madison, WI, USA).

qRT-PCR detection of DUXAP10. The real-time quantitative reverse transcription-polymerase chain reaction (qRT-PCR) was achieved by using the GoTaq qPCR Master Mix (Promega) on a Mx3005P real-time PCR system (Stratagene, La Jolla, CA, USA) following the manufacturer's instructions. Primers for DUXAP10 and actin were synthesized by Sangon Biotech (Shanghai, China). The primer sequences used in the study are listed in Table I. The data were analyzed using the $\Delta \mathrm{Ct}$ method. All results are expressed as the mean \pm SEM of the three independent experiments.

Protein extraction and western blot analysis. Western blot analysis was performed to analyze cell cycle and apoptotic protein expression. The cells were collected and lysed with cell lysis buffer for western blotting (Beyotime, Haimen,
China). The proteins (30 $\mu \mathrm{g}$ per lane) were separated on $12 \%$ SDS-polyacrylamide gels and transferred onto polyvinylidene fluoride (PVDF) membranes (Millipore, Billerica, MA, USA). Immunoblotting of the membranes was performed using the following primary antibodies: 1:1,000 rabbit anti-human phospho-Akt (Ser473), Akt, P13K-p110a, phospho-mTOR, m-TOR, PTEN, cleaved caspase-3, cleaved caspase-9, Bcl-xL, Bad, CDK4, cyclin E, p27 (Cell Signaling, Danvers, MA, USA) and 1:1,000 mouse anti-human $\beta$-actin (4A Biotech, Beijing, China). The signals were revealed after incubation with the recommended secondary antibodies (1:4,000 goat anti-rabbit IgG HRP-conjugated) (Transgen Biotech, Beijing, China) using a Bio-Rad ChemiDOX XRS+ Imaging system (Bio-Rad Laboratories, Inc., CA, USA).

RNA interference. siRNA oligos targeting DUXAP10 (si-DUXAP10) and non-targeting siRNAs (negative control, si-NC) were designed and synthesized from GenePharma (Shanghai, China). Three siRNAs were designed in our pre-experiment (DUXAP10-home-957, DUXAP10-home-773 and DUXAP10-home-1080) and the transfection effect of DUXAP10-home-957 was found to be the best. The sequences of the si-DUXAP10 and the si-NC used are listed in Table I. The sequences of the two other siRNAs are listed in Table II. The cells were cultured in 6 well-plates, and were maintained in reduced serum Opti-MEM medium (Gibco). siRNA transfections were performed with $120 \mathrm{nM}$ siRNA and $2 \mu \mathrm{l}$ (per well) Lipofectamine 2000 (Gibco) following the manufacturer's instructions. After transfection, the medium was removed and the cells were cultured in RPMI-1640 and F12-k medium supplemented with $10 \%$ fetal bovine serum.

Cell proliferation. For the cell proliferation assay, cells were plated in E-Plate 96 (ACEA Biosciences, San Diego, CA, USA), using a Real-Time Cell Analyzer (RTCA) (ACEA Biosciences) according to the manufacturer's instructions and the cell number was calculated by TC10 Automated Cell Counter (Bio-Rad Laboratories, Inc.).

Cell cycle and apoptosis. The cells were washed with PBS and fixed in $75 \%$ ice-cold ethanol at $-20^{\circ} \mathrm{C}$ overnight. After rehydrating with ice-cold PBS, the cells were stained with cell cycle staining solution (MultiSciences Co., Ltd., Beijing, China) and analyzed by flow cytometry, on a FACSCalibur flow cytometer (BD Biosciences) using CellQuest Pro software. The cells were washed in PBS and stained in Annexin V/PI apoptosis kit (MultiSciences Co., Ltd.) and the rate of cell apoptosis was also analyzed by flow cytometry on a FACSCalibur flow cytometer.

Statistical analysis. The data are presented as the mean \pm SEM The differences between groups were evaluated with two-tailed Student's t-tests using SPSS Statistics 20.0 software (IBM, Armonk, NY, USA). p $<0.05$ was considered to be statistically significant.

\section{Results}

DUXAP10 is overexpressed in BC cells. The relative expression level of DUXAP10 was evaluated in BC cells T24, 5637, E-j, TCCSUP, UM-UC-3, RT4, and normal urothelial 
Table I. Sequences of primer and siRNA $\left(5^{\prime} \rightarrow 3^{\prime}\right)$.

\begin{tabular}{lll}
\hline Name & \multicolumn{1}{c}{ Sense } & \multicolumn{1}{c}{ Antisense } \\
\hline IncRNA-DUXAP10 primer & GGCTGGAAGATTGCTTGAG & GGTAGTGTGGTGCCTCCTGT \\
Actin primer & CATGTACGTTGCTATCCAGGC & CTCCTTAATGTCACGCACGAT \\
lncRNA-DUXAP10 siRNA & GACUAUGCCUUCUGAAUAUTT & AUAUUCAGAAGGCAUAGUCTT \\
Negative control siRNA & UUCUCCGAACGUGUCACGUTT & ACGUGACACGUUCGGAGAATT \\
\hline
\end{tabular}

Table II. Sequences of siRNA $\left(5^{\prime} \rightarrow 3^{\prime}\right)$.

\begin{tabular}{lll}
\hline Name & \multicolumn{1}{c}{ Sense } & \multicolumn{1}{c}{ Antisense } \\
\hline DUXAP10-home-773 & GAGAAUGGGUCUAAAAGGAATT & UUCCUUUAGACCCAUUCUCTT \\
DUXAP10-home-1080 & CCAUAUCCUGGUAAGGCUUTT & AAGCCUUACCAUAUGGTT \\
\hline
\end{tabular}

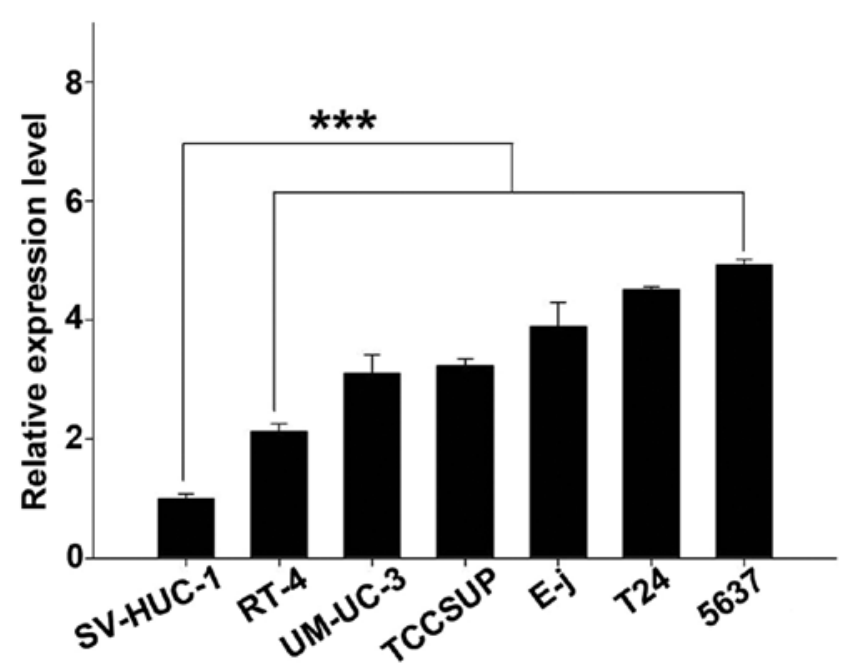

Figure 1. Expression of DUXAP10 in normal and BC cells. The expression of DUXAP10 in BC cells 5637, T24, E-j, TCCSUP, UM-UC-3 and RT4 was higher compared with SV-HUC-1. Data are shown as mean \pm SEM, $n=3$. **** $<<0.001$

cell SV-HUC-1. Comparing with SV-HUC-1, the expression of DUXAP10 is significantly upregulated in $5637(493.0 \%)$, T24 (451.3\%), E-j (389.3\%), TCCSUP (323.5\%), UM-UC-3 $(310.9 \%)$ and RT4 $(213.1 \%)$ cells $(\mathrm{p}<0.01)$ (Fig. 1), which provides evidence that DUXAP10 may play a role in BC.

Knockdown of DUXAP10 inhibits cell proliferation in human $B C$ cells. To study the biological functions of DUXAP10 in BC cells, we designed a siRNA to knock down DUXAP10 expression. As T24 and 5637 have highly expressed DUXAP10, we chose both T24 and 5637 as cell models for further investigation. After transfection with si-DUXAP10, the expression of DUXAP10 is significantly reduced comparing to transfection with si-NC ( $\mathrm{p}<0.01)$ (Fig. 2A).

Inhibition of cell proliferation was observed in T24 and 5637 cells after transfection of si-DUXAP10 at 12 and $24 \mathrm{~h}$, respectively ( $\mathrm{p}<0.01$ ) (Fig. $2 \mathrm{C}$ and D). Moreover, knockdown of DUXAP10 had no effect on cell proliferation in the SV-HUC-1 cells (Fig. 2B). These results indicate that DUXAP10 is associated with tumor cell proliferation.

The effect of DUXAP10 knockdown on cell cycle in human $B C$ cells. Knockdown of DUXAP10 significantly affected cell proliferation, so we decided to investigate the effects of DUXAP10 on the cell cycle. Flow cytometry demonstrated that DUXAP10 downregulation induced cell cycle arrest in G0/G1 in 5637 and T24 cells ( $<<0.01$ ) (Fig. 3A and B). These results indicated that the inhibitory effect on T24 and 5637 cell proliferation after knockdown of DUXAP10 may be taken through restraining the G0/G1 to $\mathrm{S}$ phase transition.

According to the results obtained from the cell cycle changes, we examined the effects of DUXAP10 on the expression levels of cyclin E, CDK4 and p27. As shown in Fig. 3C, the p27 protein level increased, while cyclin E and CDK4 protein level decreased by the knockdown of DUXAP10 in T24 and 5637 cells. These results suggest that the G0/G1 cell cycle arrest induced by knockdown of DUXAP10 was related to the downregulation of cyclin $\mathrm{E}$ and $\mathrm{CDK} 4$ and upregulation of the $\mathrm{p} 27$ protein.

Knockdown of DUXAP10 promotes apoptosis in human BC cells. To further study the biological function of DUXAP10, we tested whether knockdown of DUXAP10 increases apoptosis in T24 and 5637 cells. The proportion of apoptotic cells after cell transfection of si-DUXAP10 was higher compared with cells transfection of si-NC (23.0 vs $10.3 \%$ and 20.1 vs $12.9 \%$, respectively) in T24 and 5637 cells ( $\mathrm{p}<0.01)$ (Fig. 4A and B). These results indicate that knockdown of DUXAP10 promotes cell apoptosis.

We also performed western blotting to detect caspase activation (Fig. 4C). After knockdown of DUXAP10 increased levels of cleaved caspase-3 and -9 expression were detected in the T24 and 5637 cells (Fig. 4C). In order to further test whether DUXAP10 induced apoptosis through the activation of the mitochondrial apoptotic pathway, we detected two major proteins involved in this pathway. As shown in Fig. 4C, DUXAP10 knockdown led to an increase in the expression of 

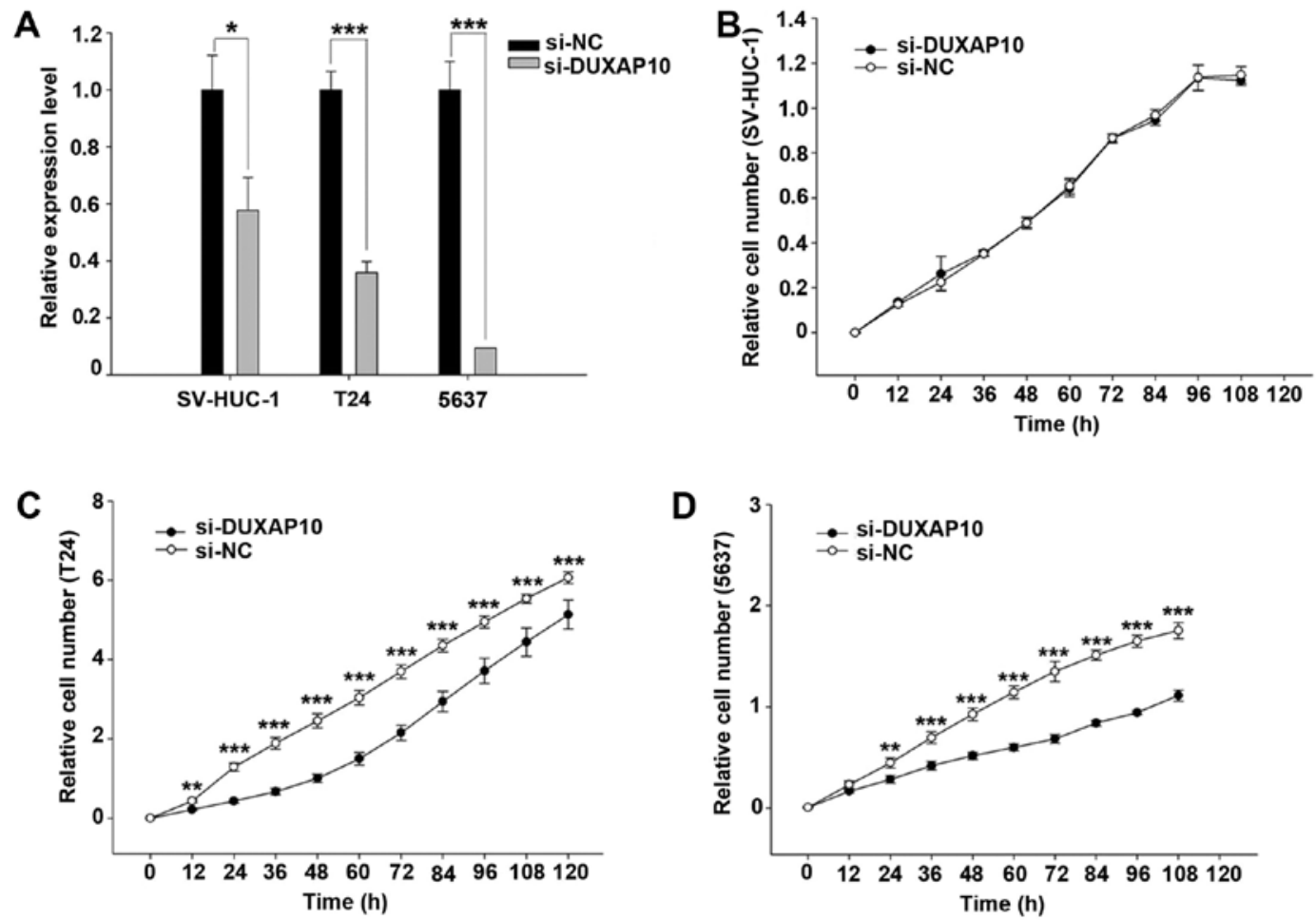

Figure 2. Cell proliferation was measured by RTCA. (A) DUXAP10 was downregulated in cells transfected with si-DUXAP10. (B) Growth curves of the human normal bladder cell line SV-HUC-1, human BC cell lines T24 (C) and 5637 (D) following si-DUXAP10 transfection. Data are presented as mean \pm SEM, n=3. ${ }^{*} \mathrm{p}<0.05,{ }^{* *} \mathrm{p}<0.01,{ }^{* * *} \mathrm{p}<0.001$.
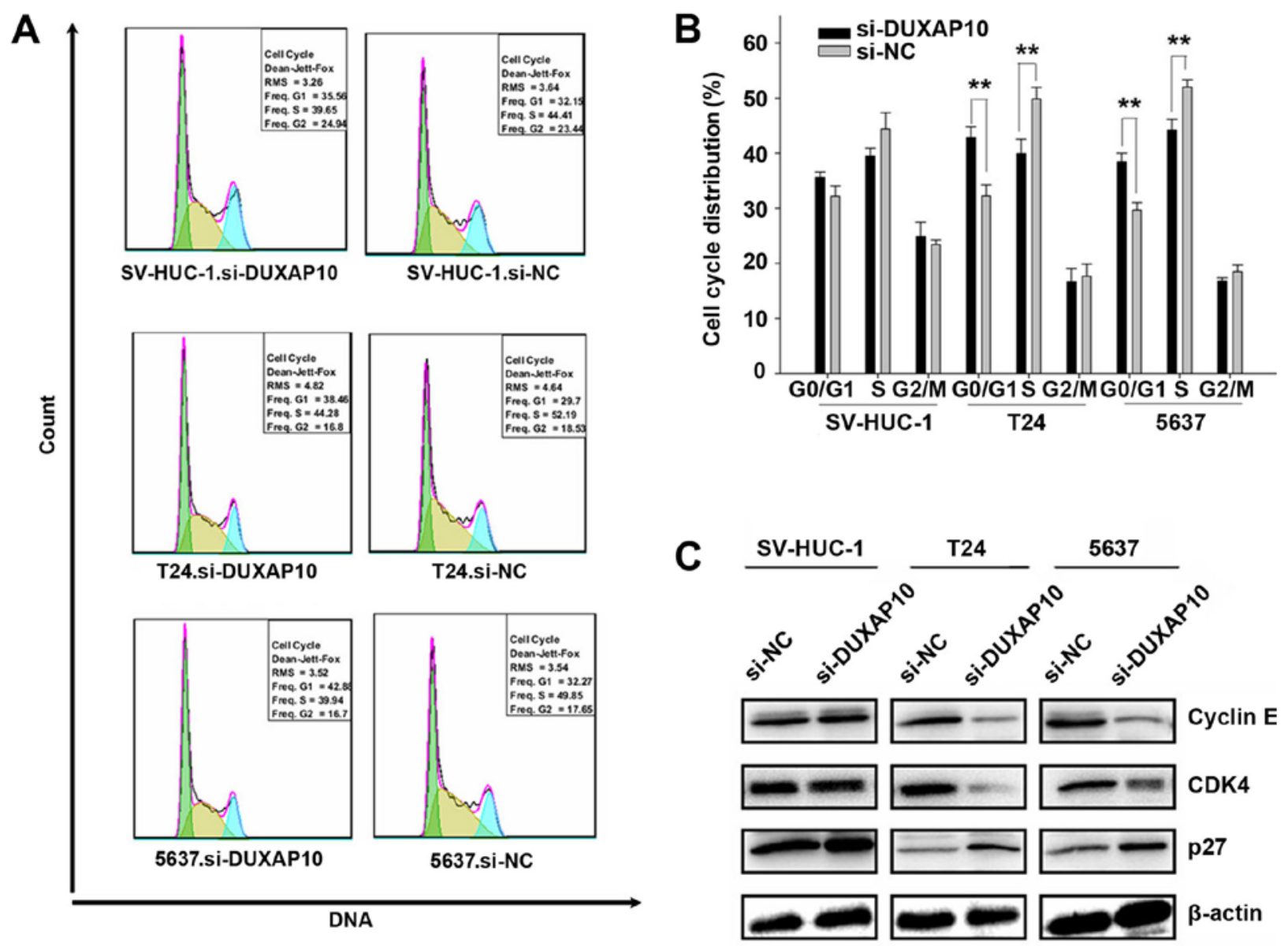

Figure 3. Knockdown of DUXAP10 induces G0/G1-phase arrest in BC cells. (A) Representative original flow cytometry results. (B) Data are presented as mean $\pm \mathrm{SEM}, \mathrm{n}=3{ }^{* *} \mathrm{p}<0.01$. (C) Western blot analysis was performed to analyze cell cycle related proteins. 
A

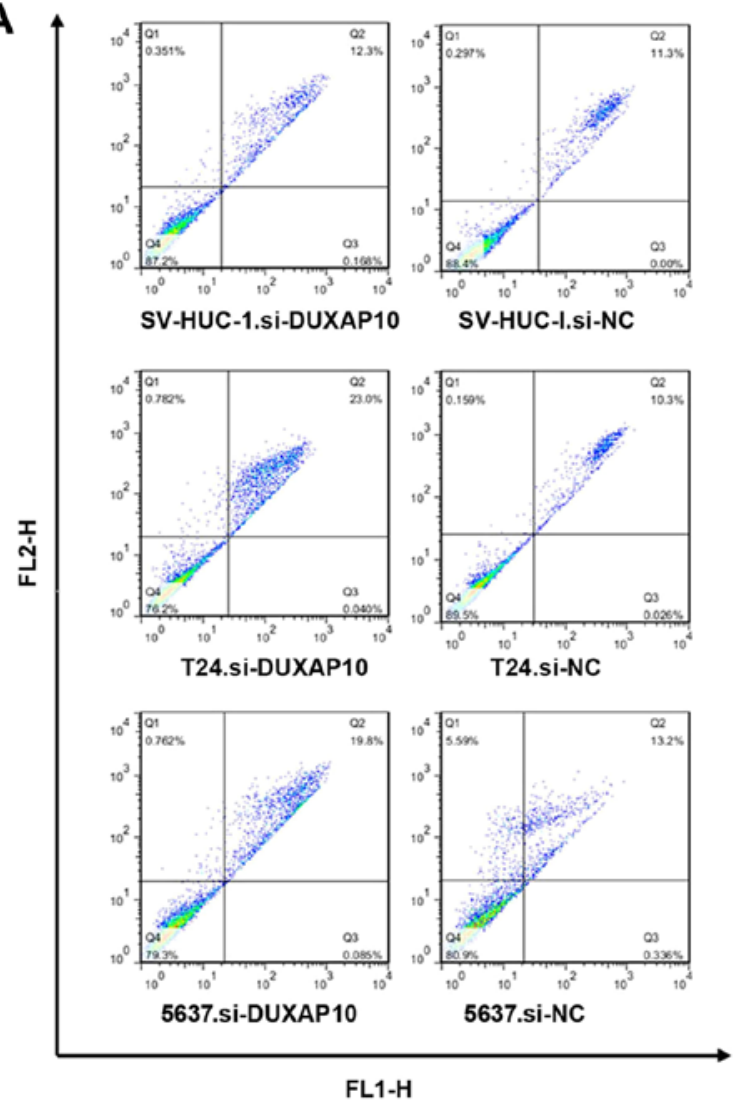

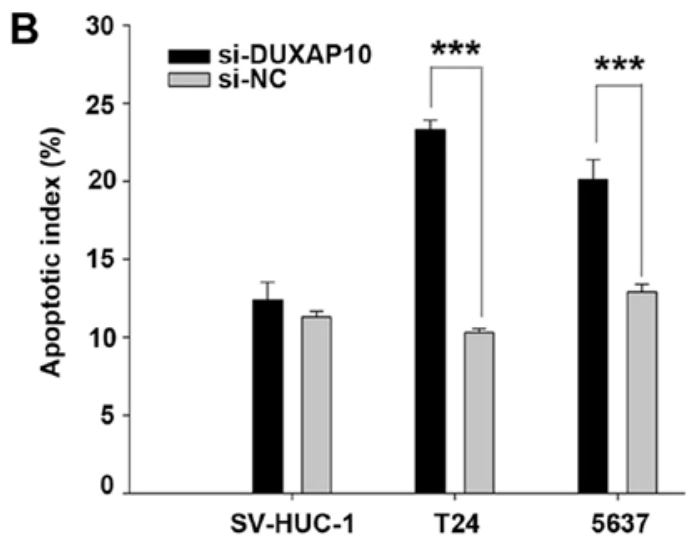

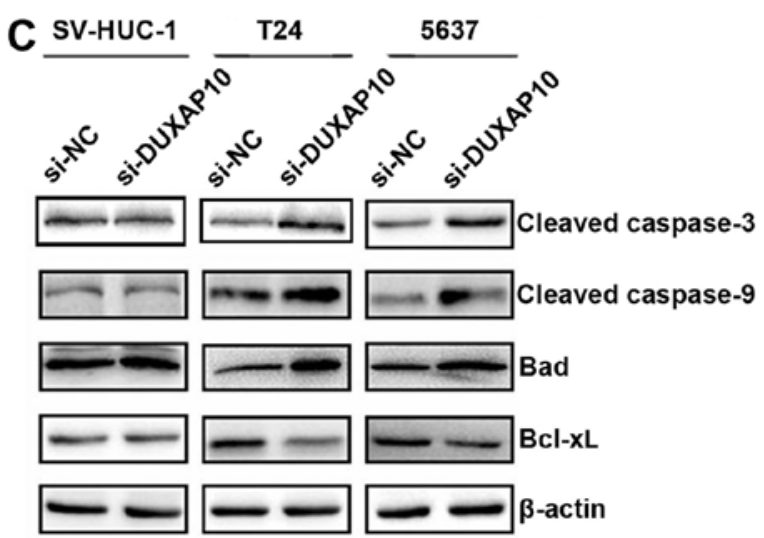

Figure 4. Knockdown of DUXAP10 induces apoptosis in BC cells. (A) Representative original flow cytometry results. (B) Data are shown as mean \pm SEM, $\mathrm{n}=3 .{ }^{* * *} \mathrm{p}<0.001$. (C) Western blot analysis of apoptosis-related proteins.

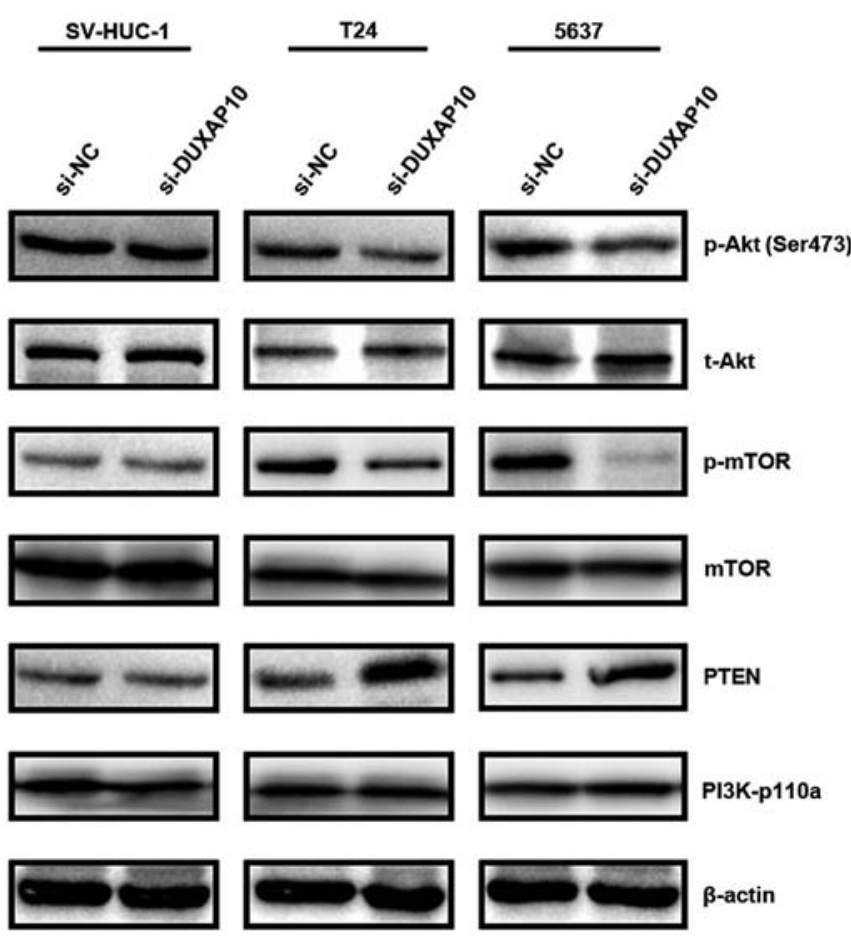

Figure 5. Western blot analysis was performed to analyze P13K/Akt/mTOR signaling pathway-related proteins. The phosphorylation levels of Akt (Ser473) and mTOR were decreased by knockdown of DUXAP10 in T24 and 5637 cells. The expression of PTEN was increased after knockdown of DUXAP10 in T24 and 5637 cells. The expressions of total Akt, P13K-p110a and mTOR showed no changes in the two cell lines. the pro-apoptotic protein Bad and a decrease in the expression of anti-apoptotic protein Bcl-xL in T24 and 5637 cells. These results indicate that the inhibition of DUXAP10 induces apoptosis through the mitochondrial apoptotic pathway.

Knockdown of DUXAP10 regulates the PI3K/Akt/mTOR signaling pathway in $B C$ cells. To explore the potential mechanisms involved in apoptosis and cell cycle arrest, we examined the effects of DUXAP10 on P13K/Akt/mTOR signaling pathways that contributes to the development of $\mathrm{BC}$. As shown in Fig. 5, the expression of PTEN was increased after knockdown of DUXAP10 in T24 and 5637 cells. Then the phosphorylation levels of Akt (Ser473) and mTOR were decreased by knockdown of DUXAP10 in T24 and 5637 cells. These results suggest that knockdown of DUXAP10 potentially exerts its antitumor function through interfering with the PI3K/AKT/mTOR signaling pathway.

\section{Discussion}

Increasing studies are focusing on the effects of lncRNAs in the diagnosis and treatment of cancers. With the development of high-throughput DNA sequencing and array-based technologies, various classes of lncRNAs have been identified (16). Currently, lncRNAs have been observed to regulate complex cellular behaviors and many of them are implicated in the development and progression of cancer (17). Dysregulation of 
lncRNAs, such as HOTAIR, H19, MALAT-1, and PCA3, have been reported as a primary feature of several human cancers including lung cancer, breast cancer, colorectal cancer and prostate cancer (18). Thus, lncRNAs may reveal novel mechanisms of tumorigenesis and progression, as well as present new targets for the purposes of cancer therapy (19).

In the present study, we found that the expression of DUXAP10 in the human BC cell lines 5637, T24, E-j, TCCSUP, UM-UC-3, and RT4 was significantly higher compared to the human normal bladder cell line SV-HUC-1 (Fig. 1). This result suggested that DUXAP10 may be associated with BC. Therefore, we designed siRNA oligos specifically targeting DUXAP10 to knock down DUXAP10 in BC cells T24, 5637 (the expression of DUXAP10 was higher in the selected cells than the others) and normal urothelial cells (Fig. 2A).

After knockdown of the expression of DUXAP10, we found that cell proliferation in BC cells T24 (12 h after transfection) and 5637 (24 h after transfection) was inhibited but had no significant effect on the SV-HUC-1 cells (Fig. 2B-D). Furthermore, apoptosis was enhanced in T24 and 5637 after the transfection of si-DUXAP10 (Fig. 4A and B). DUXAP10 knockdown increased the distribution of G0/G1 and decreased the distribution of S phase in T24 and 5637 (Fig. 3A and B). The results indicate that DUXAP10 is able to accelerate proliferation through promoting the G0/G1 to $\mathrm{S}$ phase transition and to depress apoptosis in BC cells T24 and 5637.

Alterations in the regulation of the cell cycle progression and apoptosis are frequent events in human cancers. The G1 cyclin-CDK complex cyclin E-CDK4 is required for $\mathrm{S}$ phase entry (20). CDK inhibitors (CKIs) such as $\mathrm{p} 27^{\mathrm{KIP} 1}$ binds to cyclin-CDK complexes and renders them inactive, resulting in the inhibition of cell cycle progression (21). Our study showed that the cyclin E and CDK4 levels dropped following knockdown of DUXAP10. However, p27 levels were elevated. These results suggest that DUXAP10 is associated with cell cycle progression via downregulation of cyclin $\mathrm{E}$ and CDK4 while with upregulation of p27 in human BC cells.

Apoptosis is a complex process regulated by a balance of pro-apoptotic and anti-apoptotic genes $(22,23)$. Our results demonstrated that knockdown of DUXAP10 enhances expression of cleaved caspase- 9 and cleaved caspase- 3 . The pro-apoptotic protein Bad was increased, while the anti-apoptotic protein Bcl-xL was decreased. These results suggest that knockdown of DUXAP10 induces apoptosis via dysregulation of Bad and Bcl-xL proteins.

To explore the potential mechanisms underlying the effects of DUXAP10, we evaluated the effect of DUXAP10 on the cellular signaling pathways involved in the inhibition of apoptosis and regulation of cell cycle arrest. Akt and mTOR are two downstream effectors of PI3K and involved in several cellular activities (24). Akt is activated by the phosphorylation of Thr308 and Ser473 key regulatory sites (25). The $\mathrm{PI} 3 \mathrm{~K} / \mathrm{Akt} / \mathrm{mTOR}$ pathway is a critical pathway in cancer and activation of this pathway is associated with tumor growth and therapeutic resistance $(24,26)$. In addition, PTEN is a lipid phosphatase that directly resists the activity of PI3K (27). In our study, western blot analysis showed that the phosphorylation of Akt (Ser473) and phosphorylation of mTOR were decreased by knockdown of DUXAP10 in both T24 and 5637 cells. Moreover, we observed that the expression of
PTEN was increased after knockdown of DUXAP10 (Fig. 5). Therefore, our data confirmed that DUXAP10 inhibited BC cell proliferation and induced cell apoptosis via PI3K/Akt/ mTOR signaling pathway.

To the best of our knowledge, this is the first study focusing on the analysis of DUXAP10 expression and its biological functions in BC cell lines. We found that DUXAP10 was overexpressed in the BC cell lines 5637, T24, E-j, TCCSUP, UM-UC-3 and RT4 when compared to normal bladder cells (Fig. 1). Knockdown of DUXAP10 inhibited cell proliferation and enhanced cell apoptosis in T24 and 5637 cells. Therefore, we can conclude that DUXAP10 plays a role in $\mathrm{BC}$ and we will investigate expression level of DUXAP10 in clinical samples and its relationship with clinical pathology and prognosis in our further study.

In conclusion, knockdown of DUXAP10 inhibits BC cell proliferation and increases apoptosis. This finding contributes to a better understanding of the importance of the dysregulated lncRNAs in $\mathrm{BC}$ progression, which provides a rationale for the potential development of lncRNA-based approaches for the treatment of $\mathrm{BC}$.

\section{Acknowledgements}

This study was supported by the National Natural Science Foundation of China (grant nos. 81272828 for Q.M., 31501113 for R.Y. and 81402099 for Y.L.), Zhejiang Provincial Foundation for Medical and Health Sciences (grant nos. 2016KYB263 and 2014KYB355 for Q.M. and 2017KY576 to J.F.C) and Natural Science Foundation of Ningbo (2016A610163 for X-Y.L).

\section{References}

1. Torre LA, Bray F, Siegel RL, Ferlay J, Lortet-Tieulent J and Jemal A: Global cancer statistics, 2012. CA Cancer J Clin 65: 87-108, 2015.

2. Siegel RL, Miller KD and Jemal A: Cancer statistics, 2016. CA Cancer J Clin 66: 7-30, 2016.

3. Clark PE, Spiess PE, Agarwal N, Bangs R, Boorjian SA, Buyyounouski MK, Efstathiou JA, Flaig TW, Friedlander T, Greenberg RE, et al: NCCN Guidelines Insights: Bladder Cancer, Version 2.2016. J Natl Compr Cancer Netw 14: 1213-1224, 2016

4. Babjuk M, Burger M, Zigeuner R, Shariat SF, van Rhijn BW, Compérat E, Sylvester RJ, Kaasinen E, Böhle A, Palou Redorta J, et al; European Association of Urology: EAU guidelines on non-muscle-invasive urothelial carcinoma of the bladder: Update 2013. Eur Urol 64: 639-653, 2013.

5. Abufaraj M, Gust K, Moschini M, Foerster B, Soria F, Mathieu R and Shariat SF: Management of muscle invasive, locally advanced and metastatic urothelial carcinoma of the bladder: A literature review with emphasis on the role of surgery. Transl Androl Urol 5: 735-744, 2016.

6. Witjes JA, Compérat E, Cowan NC, De Santis M, Gakis G, Lebret T, Ribal MJ, Van der Heijden AG and Sherif A: AU guidelines on muscle-invasive and metastatic bladder cancer: summary of the 2013 guidelines. Eur Urol 65: 778-792, 2014.

7. Bidnur S, Savdie R and Black PC: Inhibiting immune checkpoints for the treatment of bladder cancer. Bladder Cancer 2: 15-25, 2016.

8. Djebali S, Davis CA, Merkel A, Dobin A,Lassmann T, Mortazavi A, Tanzer A, Lagarde J, Lin W, Schlesinger F, et al: Landscape of transcription in human cells. Nature 489: 101-108, 2012.

9. Ponting CP, Oliver PL and Reik W: Evolution and functions of long noncoding RNAs. Cell 136: 629-641, 2009.

10. Kong YW, Ferland-McCollough D, Jackson TJ and Bushell M: microRNAs in cancer management. Lancet Oncol 13: e249-e258, 2012. 
11. Enokida H, Yoshino H, Matsushita R and Nakagawa M: The role of microRNAs in bladder cancer. Investig Clin Urol 57 (Suppl 1): S60-S76, 2016.

12. Chen J, Miao Z, Xue B, Shan Y, Weng G and Shen B: Long noncoding RNAs in urologic malignancies: Functional roles and clinical translation. J Cancer 7: 1842-1855, 2016.

13. Li HJ, Li X, Pang H, Pan JJ, Xie XJ and Chen W: Long noncoding RNA UCA1 promotes glutamine metabolism by targeting miR-16 in human bladder cancer. Jpn J Clin Oncol 45: 1055-1063, 2015.

14. Zhu H, Li X, Song Y, Zhang P, Xiao Y and Xing Y: Long noncoding RNA ANRIL is up-regulated in bladder cancer and regulates bladder cancer cell proliferation and apoptosis through the intrinsic pathway. Biochem Biophys Res Commun 467: 223-228, 2015.

15. Li LJ, Zhu JL, Bao WS, Chen DK, Huang WW and Weng ZL: Long noncoding RNA GHET1 promotes the development of bladder cancer. Int J Clin Exp Pathol 7: 7196-7205, 2014.

16. Zhu YP, Bian XJ, Ye DW, Yao XD, Zhang SL, Dai B, Zhang HL and Shen YJ: Long noncoding RNA expression signatures of bladder cancer revealed by microarray. Oncol Lett 7: 1197-1202, 2014.

17. Schmitt AM and Chang HY: Long noncoding RNAs in cancer pathways. Cancer Cell 29: 452-463, 2016.

18. Bartonicek N, Maag JL and Dinger ME: Long noncoding RNAs in cancer: Mechanisms of action and technological advancements. Mol Cancer 15: 43, 2016.
19. Jiang $\mathrm{C}$, Li $\mathrm{X}$, Zhao $\mathrm{H}$ and Liu $\mathrm{H}$ : Long non-coding RNAs: Potential new biomarkers for predicting tumor invasion and metastasis. Mol Cancer 15: 62, 2016

20. Sheppard KE and McArthur GA: The cell-cycle regulator CDK4 An emerging therapeutic target in melanoma. Clin Cancer Res 19: 5320-5328, 2013.

21. Bertoli C, Skotheim JM and de Bruin RA: Control of cell cycle transcription during G1 and S phases. Nat Rev Mol Cell Biol 14: 518-528, 2013.

22. Um HD: Bcl-2 family proteins as regulators of cancer cell invasion and metastasis: A review focusing on mitochondrial respiration and reactive oxygen species. Oncotarget 7: 5193-5203, 2016.

23. Gibson CJ and Davids MS: BCL-2 antagonism to target the intrinsic mitochondrial pathway of apoptosis. Clin Cancer Res 21: 5021-5029, 2015.

24. Guo H, German P, Bai S, Barnes S, Guo W, Qi X, Lou H, Liang J, Jonasch E, Mills GB, et al: The PI3K/AKT pathway and renal cell carcinoma. J Genet Genomics 42: 343-353, 2015.

25. Nitulescu GM, Margina D, Juzenas P, Peng Q, Olaru OT, Saloustros E, Fenga C, Spandidos DA, Libra M and Tsatsakis AM: Akt inhibitors in cancer treatment: The long journey from drug discovery to clinical use (Review). Int J Oncol 48: 869-885, 2016.

26. Lee JJ, Loh K and Yap YS: PI3K/Akt/mTOR inhibitors in breast cancer. Cancer Biol Med 12: 342-354, 2015.

27. Engelman JA: Targeting PI3K signalling in cancer: Opportunities, challenges and limitations. Nat Rev Cancer 9: 550-562, 2009. 